\title{
Adjusting to the COVID-19 pandemic: challenges and opportunities of frontline colorectal cancer teams in the UK
}

\author{
Rabiya Aseem ${ }^{1}$ (D) $\cdot$ Oliver Warren $^{2} \cdot$ Sarah Mills $^{2} \cdot$ Jason Smith ${ }^{1} \cdot$ Nikhil Pawa $^{1}$
}

Accepted: 20 May 2020 / Published online: 27 May 2020

(C) Springer-Verlag GmbH Germany, part of Springer Nature 2020

Dear Editor:

The World Health Organization (WHO) declared the worldwide outbreak of coronavirus disease (COVID-19) a pandemic on 11 March 2020 [1]. The first diagnosis of COVID-19 in the UK was acknowledged in early January, and as of April 28th, there have been a total of 161,145 confirmed cases and 21,678 hospital deaths [2]. Colorectal cancer (CRC) services have had to rapidly adapt to the unprecedented changes compared with standard pre-practice. Clinical teams underwent instantaneous restructuring as all junior staff were redeployed to critical care. Elective colorectal surgery was suspended to accommodate for the amassed requirement of ventilatory space. However, NHS England issued detailed guidance on the continuity of cancer services [3]. Further recommendations advocated a 'hub-and-spoke' arrangement along with a designated senior responsible officer (SRO) to form regional cancer alliances. This combined strategy would allow for effective prioritisation of urgent CRC on the basis of clinical urgency $[3,4]$.

Needless to say, caring for CRC patients poses exceptional challenges due to the unparalleled circumstances instigated by the pandemic. Frontline cancer organisations have been working overtime to implement safe management for patients with restricted resources. There is a sense of urgency amongst CRC teams to postulate prompt treatment for patients over concerns regarding cancer progression and subsequent complications. However, maintaining cancer outcomes amidst minimising infection spread in patients is an arduous dilemma. Recent reports

Rabiya Aseem

rabiya.aseem@nhs.net

1 West Middlesex University Hospital, Chelsea and Westminster NHS Foundation Trust, Twickenham Rd, Isleworth TW7 6AF, UK

2 Chelsea and Westminster Hospital NHS Foundation Trust, 369 Fulham Rd, Chelsea, London SW10 9NH, UK have proposed that CRC patients have a higher mortality from COVID-19 [5]; therefore, careful risk versus benefit assessments are being conducted for all patients.

The government introduced a widespread lockdown in the UK to reduce the spread of infection on 23 March 2020. In response, a significant proportion of colorectal face-to-face appointments, including Two-Week Wait (2WW) cancer appointments, have been successfully virtualised in an effort to protect patients and clinicians from viral exposure. Telephone consultations have been sufficient to discuss red flag symptomatology; however, these understandably do not offset clinical examinations. Thus, patients displaying high suspicion of CRC are invited to attend hospital appointments following consultant-led risk assessments. Faecal immunochemical testing (FIT) has played a pivotal role in triaging patients and streamlining referrals from primary care to CRC services. The British Society of Gastroenterology (BSG) has produced firm guidelines to limit endoscopic and virtual colonoscopies given the potential risks of aerosol generating procedures (AGP) [6]. Consultants have had the difficult task of rationing endoscopic and radiological tests for only high-priority cases. CT scanning, yet restricted, has been useful in risk stratification for suspected CRC. Patients exhibiting suspicious findings on imaging are evaluated and thus prioritised for staging procedures.

The effective prioritisation of CRC patients and subsequent management has been conceivable due to the expertise of our local multidisciplinary teams (MDTs). MDT meetings were transformed and virtualised overnight, and like many other international nations, the UK raced to adopt virtualisation to obviate the necessity for physical interaction [7]. Technical difficulties and stakeholder engagement were obvious challenges; however, these subsided quickly with increasing familiarity of videoconferencing software. Pre-pandemic oncological algorithms for the management of CRC have undergone reform, and treatment decisions are being made on a case-by-case basis [3, 4]. Risk versus benefit ratios are being employed to categorise patients for systemic therapy. Where 
possible, therapy is shortened to reduce the significant risk to patient morbidity and mortality.

Decisions regarding surgical intervention in confirmed CRC patients have always been multifaceted, and the arrival of COVID-19 has added further complexities. Initially, a 'traffic light' triage classification system was used to streamline and weigh up the urgency for surgery, depending upon symptomatology, stage of cancer and patient co-morbidities. Most recently, the 'Preventing Viral Pandemic Associated Risk of Cancer Death' (PREVAIL) criterion has been adapted to prioritise patients with CRC [8]. This pathway utilises an existing structure of NHS tumour-specific cancer MDTs to help harmonise CRC care and prevent disparities in diagnosis, surgical intervention and non-surgical treatment options. Patient outcomes are being dynamically recorded and appraised in real time to improve pandemic practices and help reduce cancer-related deaths.

The effective management of patient expectations has been a focal obstacle during this unprecedented pandemic. It is well known that patient perceptions of cancer diagnosis and treatment have profound effects on overall outcomes [9]. Thus, it is crucial to thoroughly counsel patients on treatment options and preparing them for possible sub-optimal consequences. Consent discussions for surgical procedures must include COVID-19-related complications such as severity of illness and ultimately the increased risk or mortality. Other key discussions are the need for open surgery, lower threshold for stomas, alterations of patient recovery, lack of critical care support/beds and changes to visitation of relatives that will need to be clearly communicated. Patients not eligible for imminent CRC surgery include palliative cancer diagnoses or conversely early asymptomatic cancers as per the PREVAIL criterion [8]. The central communication concerns with such patients are the progression of CRC and overall prognosis. Honest discussions and subsequent clear documentation regarding progressive disease and poor prognoses need to be practiced meticulously. All documentation, including operation notes, must be sign-posted with an indication that they coincided with the pandemic to monitor patient outcomes in comparison with previous standard practice. It is prudent that all delays in treatment, trials of pre-habilitation and oncological and surgical risks are documented to keep trails of decision-making in case of future medicolegal complications.

London hospitals have encountered significant service demands secondary to the COVID-19 pandemic. Upscaling critical care services has had a profound effect on surgical resources and availability of 'clean' COVID-free sites. CRC units have been quick to implement centralisation of services via a hub-and-spoke model approach. This allows escalation of high-priority CRC patients to surgical intervention with minimal delay. Every region has multiple spoke hospitals which streamline patients to a designated cancer hub. All accessible resources are strategically pooled, and a clean operating facility is assigned, mostly within the independent sector, incorporating a collective of surgical and anaesthetic consultants to deliver surgery. All patients are screened for COVID-19 symptoms via telephone, and viral PCR swabs along with pre-operative $\mathrm{CT}$ chest are performed to evaluate viral risk. Dual consultant-delivered procedures are conducted to ensure maximisation of theatre and reduced operating times. It is prudent that all operating surgeons adhere to strict personal protective equipment (PPE) guidelines.

The unforeseen arrival of the COVID-19 pandemic will reconfigure healthcare practice for the foreseeable future. It is crucial that $\mathrm{CRC}$ units implement urgent strategies to reduce the pending clinical backlog. The enduring pandemic presents a valuable opportunity for all colorectal surgeons to revaluate their practices to maximise efficiency, clinical effectiveness and improve patient care. Virtualisation of current working patterns, especially CRC outpatient clinics, has demonstrated compliance amongst healthcare professionals and patients, thus showing real promise for the future. It is vital that we explore and reinforce the sustainability of virtual healthcare and its overall impact on both clinicians and patients.

The assessment and management of 2WW CRC referrals have undergone substantial transformation during this pandemic. Consultant-led triaging has resulted in enhanced risk assessments and streamlined decision-making to avoid dissemination of unnecessary investigations. Tighter inclusion criterion within MDTs needs maintaining, and tracking systems should be frequently reviewed to enhance surveillance of CRC patients. Furthermore, dynamic consultant-led assessments are fundamental for the continual stratification of CRC patients and subsequent planning of investigations. The benefit of dual consultant operating is apparent, and long-term considerations to cement such practice should be re-evaluated, acknowledging prospective effects on patient care and surgical training.

It is mandatory that all modifications to pre-pandemic practices are objectively recorded and assessed for clinical effectiveness and patient-related outcomes. Pristine documentation regarding deviations from pre-pandemic practice is compulsory to ensure retrospective trailing of decisions. Multiple international and national organisations have developed research collaboratives assessing clinical parameters of surgical patients and consequent outcomes during the COVID-19 pandemic. It is prudent that all CRC units assist with research collaborations, as such data will be critical for optimising the future management of patients.

It is inevitable that the pandemic will have significant effects on staff's well-being and mental health. Burnout will be a major concern amongst colleagues due to changes in working patterns, increasing workload and redeployment to nonsurgical specialities. Identifying vulnerable individuals and providing support for one another will be crucial to counteract mental health issues. Colorectal team leaders are required to 
encourage junior members to seek help and allow for open means of communication.

The provision of colorectal surgery will continue to be unparalleled, and CRC patients will be relying on surgeons for support during this extraordinary time. Exemplary leadership qualities are required to keep our systems and teams resilient. As clinicians, we need to live up to the essence of delivering patient-centred care and optimise all relevant care pathways. It is our duty to rise to the occasion and instrumentalise organisational changes to effectively adapt to the chaos caused by the COVID-19 pandemic for the ultimate benefit of our patients.

\section{Compliance with ethical standards}

Conflict of interest The authors declare that they have no conflict of interest.

\section{References}

1. WHO (2020) Rolling updates on corona virus disease (COVID-19) [Website]. Available at: https://www.who.int/emergencies/diseases/ novel-coronavirus-2019/events-as-they-happen. Accessed 27th April 2020

2. UK Government (2020) Corona virus (COVID-19) in the UK [Website]. Available at: https://coronavirus.data.gov.uk/\#countries. Accessed 28th April 2020

3. Academy of Medical Royal Colleges (2020) Clinical guide for the management of essential cancer surgery for adults during the coronavirus pandemic. NHS England and NHS Improvement. https://www.england.nhs.uk/coronavirus/wp-content/uploads/sites/ 52/2020/04/C0239-Specialty-guide-Essential-Cancer-surgery-andcoronavirus-v1-70420.pdf Accessed on 20th April 2020

4. Walsh $\mathrm{C}$ et al (2020) Considerations for multidisciplinary management of patients with colorectal cancer during the COVID-19 pandemic. The Association of Coloproctology of Great Britain and Ireland. https://www.acpgbi.org.uk/content/uploads/2020/03/ ACPGBI-statement-on-CRC-treatment-during-COVID-19-FINAL. pdf Accessed on 20th April 2020

5. Liang W, Guan W, Chen R, Wang W, Li J, Xu K, Li C, Ai Q, Lu W, Liang H, Li S, He J (2020) Cancer patients in SARS-CoV-2 infection: a nationwide analysis in China. Lancet Oncol 21:335-337. https://doi.org/10.1016/S1470-2045(20)30096-6

6. British Society of Gastroenterology (2020) Joint ACPGBI, BSG and BSGAR considerations for adapting the rapid access colorectoral cancer pathway during COVID-19 pandemic [Website]. Available at https://www.bsg.org.uk/covid-19-advice/covid-19-advice-forhealthcare-professionals/joint-acgbbi-bsg-and-bsgar-considerationsfor-adapting-the-rapid-access-colorectoral-cancer-pathway-duringcovid-19-pandemic/. Accessed 20th April 2020

7. Webster P (2020) Virtual health care in the era of COVID-19. Lancet. 395:1180-1181. https://doi.org/10.1016/S0140-6736(20) 30818-7

8. Pan London Cancer Hubs (2020) Preventing viral pandemic associated risk of cancer death: the PREVAIL pathway. National Health Service (NHS)

9. Hofman M, Morrow GR, Roscoe JA, Hickok JT, Mustian KM, Moore DF, Wade JL, Fitch TR (2004) Cancer patients' expectations of experiencing treatment-related side effects. Cancer. 101:851-857. https://doi.org/10.1002/cncr.20423

Publisher's note Springer Nature remains neutral with regard to jurisdictional claims in published maps and institutional affiliations. 